\title{
Digital Health, Telehealth, and Primary Care Post-COVID: A Discussion with Kim Boyd and Joe Kvedar, Moderated by Benjamin Rosner
}

\author{
Benjamin I. Rosner ${ }^{a} \quad$ Masha Morozov $^{b}$ Anna Andoni ${ }^{b}$ \\ a University of California San Francisco, San Francisco, CA, USA; bNODE. Health, New York, \\ NY, USA
}

Keywords

Telehealth · Primary care $\cdot$ COVID

\section{Introduction}

The SARS-CoV-2 pandemic has been a catalyst for the advancement and adoption of telehealth. Utilized as an alternative method of delivering care that enables healthcare providers to interact with patients remotely, telehealth has helped ease the burden of in-person clinical care throughout the pandemic. The natural experiment in telehealth created by the pandemic has tested both the limits and the capabilities of telehealth and offers many learning opportunities that can be applied in the future to nonurgent, remote, synchronous healthcare delivery.

On August 31, 2020, NODE.Health, a 501(c)3 nonprofit organization dedicated to accelerating the adoption of digital technology by creating and promoting best practices in digital evidence and transformation, hosted a webinar about how telehealth and primary care have

The NODE.Health webinars are part of a series of presentations, discussions, sessions and other events open to the public and to NODE.Health's membership that are intended to bring expert perspectives on topics related to evidence, digital health, and innovation. These sessions continue during a dedicated week as part of NODE.Health's Digital Medicine Conference scheduled to be held on December 7-11, 2020. While NODE.Health supports the causes of many of its speakers, NODE. Health does not necessarily endorse any product or belief mentioned in these presentations or beyond. Elements of the webinar have been redacted or summarized for clarity. 
been affected and shaped by the SARS-CoV-2 pandemic [1]. The session was moderated by Dr. Benjamin Rosner, MD, PhD, and included guest panelists Dr. Kim Boyd, MD, and Dr. Joseph Kvedar, MD.

Dr. Rosner is an associate professor and hospitalist at the University of California San Francisco, a digital health researcher at the Center for Clinical Informatics and Improvement Research, and the previous chief medical information officer of a leading digital health company. He serves as the NODE.Health scientific chair. Dr. Boyd is a Stanford-trained physician with over a decade of experience working as a physician leader in health startups, a current consultant at Remedy Health, and the previous medical director for next-generation primary care organizations such as One Medical, NURX, and Galileo. She has expertise in virtual medicine, clinical systems and operations, and integrative wellness. Dr. Boyd currently advises digital health companies on cross-functional program development, clinical operations to build scalable systems, and clinical strategy. Dr. Kvedar is the president of the American Telemedicine Association (ATA), vice president of Connected Health at Partners Healthcare, co-chair of the American Medical Association's (AMA) Digital Medicine Payment Advisory Group, senior advisor of Virtual Care at Mass General Brigham, professor of dermatology at Harvard Medical School, and editor in chief of npj Digital Medicine, a Nature Research journal. Dr. Kvedar is also the author of 2 books on digital health; The Internet of Healthy Things (2015), and The New Mobile Age: How Technology Will Extend the Healthspan and Optimize the Lifespan (2017).

\section{Definitions of Telehealth}

To set the context for the webinar, Drs. Boyd and Kvedar described telehealth as care delivered through technology when a patient and a healthcare provider are not physically in the same space. Dr. Kvedar further elaborated that telehealth may be synchronous when a patient and a healthcare provider are connected in real-time via telephone or video encounter or asynchronous when an "encounter" is instead a sequence of messages exchanged back and forth over time.

\section{The History of Telehealth}

Dr. Boyd shared that telehealth got its start in the 1940s when phone lines were first used to transmit medical information. The University of Nebraska and the Veterans Affairs Administration adopted telephonic telehealth in the 1960s as a means to provide healthcare in "care deserts" and rural areas lacking access to in-person care. By 2018, even with the advent of video visits, telehealth was still considered the exception rather than the rule, with only about $11 \%$ of consumers having used telehealth [2].

Over the last 27 years, Dr. Kvedar described the work of the American Telemedicine Association (ATA) as "the only organization completely focused on accelerating the adoption of telehealth" and working to "change the way the world thinks about healthcare." He described the ATA as an organization comprised of hospitals and health systems, telehealth solution providers, and special interest groups that have been at work advancing telehealth practice guidelines and policy, as well as advocating for telehealth at the federal and state levels.

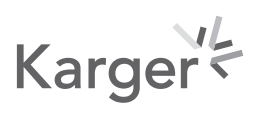




\section{The Coronavirus Pandemic as an Unprecedented Telehealth Catalyst}

Although telehealth had seen slow but steady growth before 2020, the arrival of the SARS-CoV-2 virus in the USA in early 2020 served as a catalyst to overcome many of the activation energy barriers that had previously limited its adoption. Nearly overnight, facilitated by more than 31 regulatory changes, over $46 \%$ of consumers had become telehealth users. As Dr. Kvedar indicated, telehealth had become a household term, and we had successfully brought the doctor's office into the home. Although COVID assessment and monitoring became a clear telehealth use case, those engaged in mental health, those leveraging remote patient monitoring, and those using a variety of other healthcare services also became beneficiaries. But Dr. Boyd also cautioned about the hype getting ahead of Hippocrates; "first do no harm." She stated that the rapid transition to telehealth affords us an opportunity to redefine and reshape healthcare so that we are not merely taking what would have happened in a traditional office visit and trying to put it into a virtual forum, but rather start asking new questions and leveraging new technologies and approaches. In this way, she asserted that tragedy could be flipped into an opportunity to transform healthcare.

\section{Regulatory Changes that Opened the Telehealth Floodgates}

The COVID-19 public health emergency, as described by Dr. Kvedar, opened the gates on regulatory restrictions that had previously hampered telehealth's growth. These changes included for example, sweeping allowances in Medicare reimbursement for telehealth services, broadening the types of healthcare providers that could bill for telehealth, relaxing the originating site restrictions that previously only permitted reimbursement for telehealth encounters that typically originated with a patient using telehealth from a rural clinic setting (rather than from a patient's home), relaxing enforcement of the Health Insurance Portability and Accountability Act, and creating interstate licensure compacts that permitted physicians in one state to provide telehealth services to patients in another.

Furthermore, Dr. Boyd mentioned that these relaxations alleviated business and operational barriers, making it more affordable for health systems to license their workforces in single rather than multiple states and eliminating the historical disparity in which telehealth services had been reimbursed at lower rates than in-person services. Despite Medicare's early move to relax reimbursement restrictions, private payers, according to Dr. Kvedar, remain the wild card, as it is unclear how long they may continue to follow suit. Dr. Kvedar also stated that telehealth could lead to duplicative charges for related or overlapping encounters, posing the questions, "Is telehealth additive or substitutive?" and "How do you manage increased access and keep costs in check?" For providers, he brought up the potential to streamline infrastructure costs and to rethink overhead calculations and workforce deployment costs.

While most of these changes suddenly created operational and reimbursement opportunities to deploy telehealth services, Dr. Kvedar noted that these relaxations remain temporary under the public health emergency, and without ongoing legislative efforts they will expire when the public health emergency is declared over.

\section{Indications and Contraindications for Telehealth}

With huge portions of the population now using telehealth, the conversation also turned to the relative safety and efficacy of telehealth. The panelists shared their beliefs that, in order for care to be delivered safely via telehealth, not only should providers of different levels of

\section{Karger's}


training (physicians, nurses, physician assistants, etc.) be provided with the appropriate ancillary tools they need to do their respective jobs safely via telehealth, but also patients and providers alike should understand certain limitations of the telehealth encounter. Not only should telehealth not be an expected encounter type for a patient with crushing chest pain, for example, but without vital signs and the traditional physical exam to aid the healthcare professional, patients should understand that telehealth may carry more diagnostic uncertainty. Dr. Kvedar pointed out that encounters for chronic condition follow-up, some urgent care scenarios, and mental health seem particularly well suited for telehealth. However, telehealth encounters may be more challenging for the development of new therapeutic relationships between patients and healthcare providers who have not yet met in person. He emphasized that learning "webside manner," including looking at the camera and using upper body language would become increasingly important in fostering telehealth relationships. However, as Dr. Boyd pointed out, if the expectations of both parties are different from what has historically been the case for in-person encounters, then high frequency incremental "microencounters," either synchronous or asynchronous, might, in fact, create more opportunity for patient-provider connection.

\section{The Digital Divide}

In bringing up a key point for understanding telehealth equity, Dr. Rosner stated that we know that there is a continued digital divide in the USA and asked the panelists how we can continue to build access for communities that are either lacking broadband or lacking the hardware to access telehealth. Dr. Kvedar responded that there is no easy solution to the problem of accessibility, and it remains an enormous challenge. However, during the public health emergency, payers were also reimbursing physicians for audio-only interactions through telephone visits, which can be an important medium if used thoughtfully. Audio communication may be the most immediate means at our disposal of crossing the digital telehealth divide. To further improve access, however, we must push for future stimulus packages that include allocations for universal broadband.

\section{Opportunities and Conclusion}

Drs. Boyd and Kvedar felt that telehealth is here to stay, but that ongoing legislative efforts must ensure the longevity of the regulatory changes that were temporarily put into play. Dr. Boyd pointed out that Q2 of 2020 was a banner year for telehealth investments, but the industry must be thoughtful with respect to what it hopes to accomplish with these investments. So many other service industries, including transportation and food delivery, have fundamentally changed their technology and business models, and the pandemic forced healthcare, typically a risk-averse industry, to rapidly adapt.

Finally, both panelists felt that there were many opportunities for telehealth through visit add-ons that will augment the encounter. These include home devices, home testing, remote patient monitoring, and digital biomarkers derived from smartphone apps that can, for example, determine the cause of a cough by interpreting the sound of the cough itself, or apps that can predict the onset of a sudden cardiac event based on the sound of the patient's voice.

Telehealth is an evolving, and increasingly important modality of healthcare delivery. While it has demonstrated success in scaling itself into current care paradigms, much is still to be done legislatively, from a health equity standpoint, and in solidifying the vision of how it will be leveraged in the healthcare delivery system of the future.

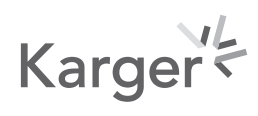




\section{Acknowledgement}

We thank Dr. Kim Boyd and Dr. Joseph Kvedar for their expert webinar contributions.

\section{Conflict of Interest Statement}

B.I.R. is a consultant to NODE.Health and declares equity interest in Infermedica. A.A. and M.M. are employees of NODE.Health.

\section{Funding Sources}

NODE.Health is a 501(c)3 that receives sponsorship funding from technology companies in support of the NODE.Health mission. Sponsors were highlighted at the end of the webinar in appreciation for their support.

\section{Author Contributions}

M.M. undertook initial transcription of the webinar. A.A. adapted the transcription for clarity. B.I.R. provided oversight, restructured the content into thematic areas, and performed final-version editing.

\section{References}

1 Rosner B, Kvedar J, Boyd K. Digital health, telehealth, and primary care post-COVID. NODE.Health. [2020 Aug 31]. Available from https://nodehealth.org/2020/10/19/open-webinar-digital-health-telehealth-andprimary-care-post-covid/

2 Bestsennyy 0, Gilbert G, Harris G, Rosy J. Telehealth: A quarter-trillion-dollar post-COVID-19 reality? [cited 2020 Oct 16]. Available from: https://www.mckinsey.com/industries/healthcare-systems-and-services/ourinsights/telehealth-a-quarter-trillion-dollar-post-covid-19-reality 